\title{
Nodule de Sour Marie-Joseph : quand le nombril révèle un cancer
}

\author{
Sister Marie-Joseph's Nodule: when the Umbilicus Reveals a Cancer
}

\author{
K. Shiraishi · M. Marcand $\cdot$ S. Muller \\ Reçu le 5 avril 2018; accepté le 3 juin 2018 \\ (C) SFMU et Lavoisier SAS 2018
}

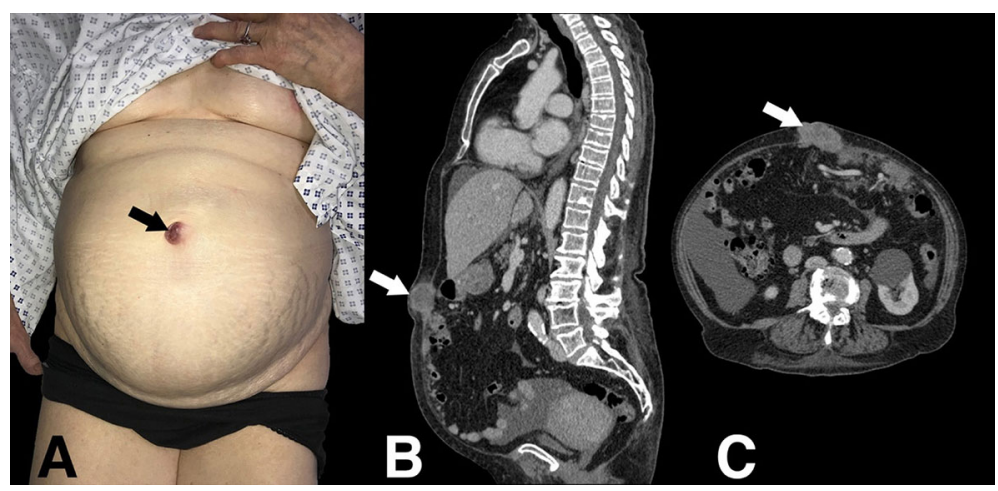

Fig. 1 Nodule désigné par une flèche sur une photographie de face, avec autorisation de la patiente (A), sur une coupe scanographique sagittale (B) et sur une coupe scanographique axiale (C). La masse hétérogène est située entre l'estomac et l'angle colique gauche

Une patiente de 80 ans hypertendue et diabétique de type 2 a été adressée aux urgences pour une tuméfaction douloureuse de l'ombilic évoluant depuis quelques semaines. À son arrivée aux urgences, la température était à $36,5^{\circ} \mathrm{C}$, la pression artérielle à $130 / 80 \mathrm{mmHg}$, la fréquence cardiaque à $85 \mathrm{bpm}$, la saturation en oxygène à $92 \%$. L'examen clinique retrouvait une tuméfaction ombilicale d'aspect violacé, infiltrée et douloureuse à la palpation (Fig. 1). La percussion abdominale révélait une matité des flancs en faveur d'une ascite. Les bruits hydroaériques étaient faiblement perceptibles. La bandelette urinaire était négative. Le bilan biologique retrouvait un syndrome inflammatoire avec des leucocytes à $20 \mathrm{~g} / \mathrm{l}$ et une CRP à $300 \mathrm{mg} / \mathrm{ml}$. Le scanner abdominopelvien a mis en évidence une masse tissulaire ombilicale associée à une masse hétérogène entre l'estomac et l'angle colique gauche, avec la présence d'ascite. L'analyse anatomopathologique de la masse et du nodule ombilical concluait à des métastases d'un adénocarcinome mucineux à différenciation intestinale. Sœur MarieJoseph (1856-1939), première assistante du chirurgien William J. Mayo (cofondateur de la Mayo Clinic), avait fait l'association entre les tumeurs ombilicales et les cancers viscé-

\footnotetext{
K. Shiraishi $(\varangle) \cdot$ M. Marcand $\cdot$ S. Muller

Service des urgences, centre hospitalier Jura Sud,

55, rue du Docteur-Jean-Michel, CS 50364,

F-39016 Lons-le-Saunier cedex, France

e-mail : kenji.shiraishi@hopitaux-jura.fr
}

raux avancés. Sir Hamilton Bailey lui rendit hommage en attribuant son nom à la métastase cutanée ombilicale de cancer abdominopelvien [1]. Un à $3 \%$ des cancers abdominopelviens métastasent à l'ombilic : principalement les cancers ovariens (34\%), colorectaux (12\%), de l'endomètre (12\%), de l'estomac $(9 \%)$ et du pancréas ( $8 \%$ ) [2]. Le nodule est généralement douloureux, ferme et induré, parfois fissuré ou ulcéré. D'apparition tardive, il signe une pathologie néoplasique avancée avec une survie moyenne de 11 mois [2]. Les diagnostics différentiels sont l'endométriose ombilicale, les granulomes pyogènes ou à corps étranger, l'hémangiome, une localisation ombilicale d'une maladie de Crohn ou un mélanome. L'étude anatomopathologique est essentielle au diagnostic. Le mode d'extension se fait surtout par contiguité à partir du péritoine, par voie veineuse, lymphatique ou par des résidus embryonnaires (ligament rond, ouraque, vestige de l'artère vitelline) [2]. En conclusion, le nodule de Sœur MarieJoseph est rare, mais caractéristique. Sa reconnaissance peut permettre le diagnostic de cancer abdominopelvien avancé.

\section{Références}

1. Jacques J, Mesturoux L, Vong C, et al (2014) Un nodule ombilical. Rev Med Interne 35:73-4

2. Dubreuil A, Dompmartin A, Barjot P, et al (1998) Umbilical metastasis or Sister Mary-Joseph's nodule. Int J Dermatol 37:7-13 